\title{
New Predictive Parameters of Bell's Palsy: Neutrophil to Lymphocyte Ratio and Platelet to Lymphocyte Ratio
}

\author{
Doğan Atan ${ }^{1}$, Aykut İkincioğullar1 ${ }^{1}$, Sabri Köseoğlu ${ }^{2}$, Kürşat Murat Özcan ${ }^{1}$, \\ Mehmet Ali Çetin ${ }^{1}$, Serdar Ensari ${ }^{1}$, Hüseyin Dere ${ }^{1}$ \\ ${ }^{1}$ Department of Otorhinolaryngology, Head and Neck Surgery, Ankara Numune Training and Research Hospital, Ankara, Turkey \\ ${ }^{2}$ Tarsus State Hospital, Mersin, Turkey
}

Background: Bell's palsy is the most frequent cause of unilateral facial paralysis. Inflammation is thought to play an important role in the pathogenesis of Bell's palsy.

Aims: Neutrophil to lymphocyte ratio (NLR) and platelet to lymphocyte ratio (PLR) are simple and inexpensive tests which are indicative of inflammation and can be calculated by all physicians. The aim of this study was to reveal correlations of Bell's palsy and degree of paralysis with NLR and PLR.

Study Design: Case-control study.

Methods: The retrospective study was performed January 2010 and December 2013. Ninety-nine patients diagnosed as Bell's palsy were included in the Bell's palsy group and ninety-nine healthy individuals with the same demographic characteristics as the Bell's palsy group were included in the control group.
As a result of analyses, NLR and PLR were calculated.

Results: The mean NLR was 4.37 in the Bell's palsy group and 1.89 in the control group with a statistically significant difference $(\mathrm{p}<0.001)$. The mean PLR was 137.5 in the Bell's palsy group and 113.75 in the control group with a statistically significant difference $(p=0.008)$. No statistically significant relation was detected between the degree of facial paralysis and NLR and PLR.

Conclusion: The NLR and the PLR were significantly higher in patients with Bell's palsy. This is the first study to reveal a relation between Bell's palsy and PLR. NLR and PLR can be used as auxiliary parameters in the diagnosis of Bell's palsy.

Keywords: Bell's palsy, inflammation, lymphocytes, neutrophils, platelets
Bell's palsy (BP) is the most frequent cause of unilateral facial paralysis at a rate of $60-75 \%$ (1). It may appear in all stages of life, most often heals completely and may recur with various severity in $15 \%$ of patients $(2,3)$. The pathogenesis of $\mathrm{BP}$ is not clear; however, inflammation and viral infections are thought to play an important role (4). As a result of inflammation, edema occurs in the fallopian tubes, especially in the labyrinth segment (5).
A hemogram of peripheral blood can be used to determine neutrophil to lymphocyte ratio (NLR) and platelet to lymphocyte ratio (PLR). PLR was found to be high in several peripheral vascular diseases, coronary artery diseases and some gynecological and hepatobiliary malignancies and was associated with poor prognosis. NLR may increase in systemic inflammation, some gynecological and gastrointestinal cancers and some cardiovascular diseases (6-8).

Address for Correspondence: Dr. Doğan Atan, Department of Otorhinolaryngology, Head and Neck Surgery, Ankara Numune Training and Research Hospital, Ankara, Turkey

Phone: +90 5555151208 e-mail: doganatan@gmail.com

Received: 17.07.2014 Accepted: 03.12.2014 • DOI: 10.5152/balkanmedj.2015.15456

Available at www.balkanmedicaljournal.org

Cite this article as:

Atan D, İkincioğulları A, Köseoğlu S, Özcan KM, Çetin MA, Ensari S, et al. New predictive parameters of Bell's palsy: neutrophil to lymphocyte ratio and platelet to lymphocyte ratio.

Balkan Med J 2015;32:167-70. 
In a recent study, NLR was found to be high in BP (9). However, there have not been any studies about PLR values in BP. Therefore, the aim of the present study was to reveal the relationship between NLR and PLR in patients with BP.

\section{MATERIALS AND METHODS}

The study was carried out in the Ear, Nose and Throat Clinic of our hospital between January 2010 and December 2013. Ethical approval was obtained from the Institutional Review Board (Approval Number 246/2014). All participants were volunteers and provided written informed consent. Ninetynine patients diagnosed as BP were assigned into the Bell's palsy group (BP group) and 99 healthy individuals were assigned into the control group. The demographic features of the study and control groups were similar. The patients diagnosed with conditions leading to facial paralysis other than BP such as trauma, Ramsey-Hunt syndrome and tumoral masses were excluded. Those patients diagnosed as BP were divided into groups based on severity of their facial paralysis according to the House-Brackmann (HB) grading system. Fifty-three patients had grade 2 paralysis, 32 patients had grade 3 paralysis, 11 patients had grade 4 paralysis and 3 patients had grade 5 paralysis. Patients with systemic diseases such as uncontrolled diabetes mellitus, uncontrolled hypertension, acute coronary artery disease, active connective tissue disorder, vasculitis, inflammatory bowel disease, chronic renal failure and chronic liver failure were excluded. Complete blood count (CBC), blood biochemistry analysis including blood lipids, thyroid hormone levels, hepatitis markers and temporal MR used for the differential diagnosis of cranial pathologies, were carried out in all of the patients at admission.

CBC was determined (Sysmex WE-2100; Sysmex, Miundelein, IL, USA) and based on CBC, erythrocyte, leukocyte, neutrophil, lymphocyte and thrombocyte counts were used to determine PLR and NLR.

Leukocyte, neutrophil, lymphocyte and platelet counts, NLR and PLR were compared between the Bell's palsy and control groups. Whether there was a difference in NLR and PLR between degrees of paralysis according to HB grading system was also determined.

\section{Statistical analysis}

Descriptive statistics were presented as mean \pm standard deviation, and the categorical variables were presented as the number of the cases and \%. The comparison of differences in age, gender and complete blood count parameters between the patient and control groups was performed using a t-test. The importance of the difference in the NLR and PLR be-
TABLE 1. Patients' age and gender distribution

\begin{tabular}{lcccc}
\hline & & BP Group & Control Group & p value \\
\hline Age & & $47.84 \pm 16.94$ & $44.22 \pm 8.64$ & 0.6 \\
Gender & Male & 46 & 54 & 0.258 \\
& Female & 53 & 45 & \\
\hline \multicolumn{2}{l}{ BP: Bell's Palsy } & & &
\end{tabular}

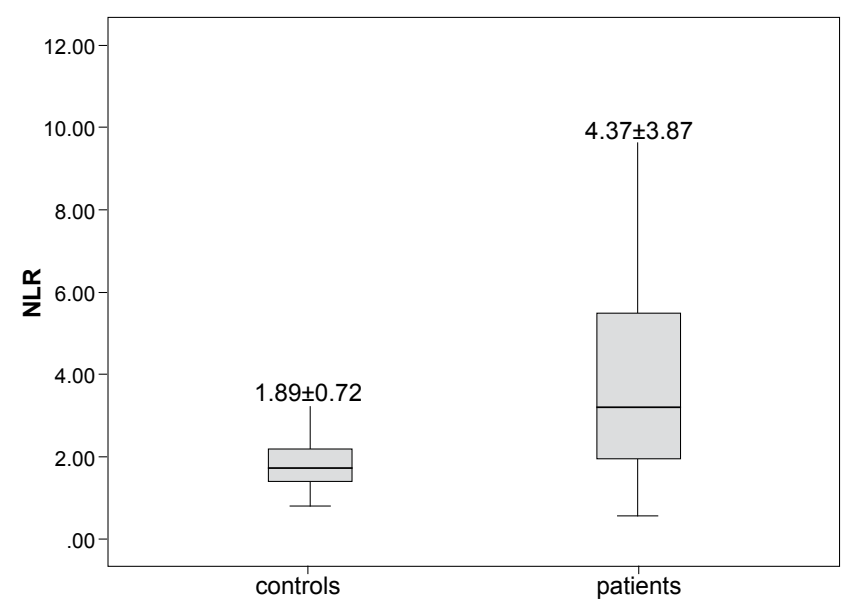

FIG. 1. The mean neutrophil to lymphocyte ratio of the patients with Bell's palsy and the control group.

tween the four groups was analyzed with the Kruskal Wallis test. $\mathrm{p}<0.05$ value was considered significant. The Statistical Package Program for Social Sciences (SPSS) for Windows, version 21.0 (SPSS Inc., Chicago, IL, USA) was used for statistical analysis.

\section{RESULTS}

The BP group included 46 males (46.5\%) and 53 (53.5\%) females and the control group included 54 (54.5\%) males and $45(45.5 \%)$ females. The mean age was $47.84 \pm 16.94$ years in the BP group and $44.22 \pm 8.64$ years in the control group. There was no statistically significant difference in age and gender between the groups $(p=0.6, p=0.258)$ (Table 1$)$.

The mean NLR was $4.37 \pm 3.87$ in the BP group and $1.89 \pm 0.72$ in the control group. It was statistically significantly higher in the BP group $(\mathrm{p}<0.001)$ (Figure 1).

The mean PLR was 137.50 \pm 81.04 in the BP group and $113.75 \pm 35.83$ in the control group. It was statistically significantly higher in the BP group $(\mathrm{p}=0.008)$ (Figure 2).

The mean leukocyte count was $11.13 \pm 4.56$ in the BP group and $7.33 \pm 1.42$ in the control group. It was statistically significantly higher in the BP group $(\mathrm{p}<0.001)$. The mean neutrophil count was $8.07 \pm 4.24$ in the BP group and $4.22 \pm 1.07$ in the control group. It was statistically significantly higher in the 


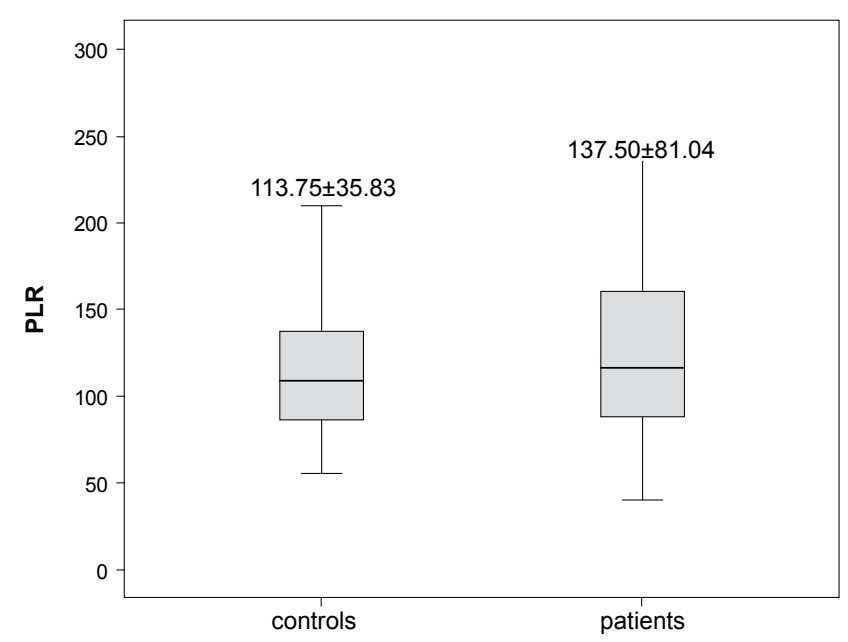

FIG. 2. The mean platelet to lymphocyte ratio of the patients with Bell's palsy and the control group.

BP group ( $\mathrm{p}<0.001)$. However, differences in lymphocyte and platelet counts were not statistically significant between the groups (Table 2). NLR and PLR values are shown according to the degree of BP disease in Table 3.

\section{DISCUSSION}

The incidence of Bell's palsy (BP), also called idiopathic peripheral facial palsy, is about $15-30$ cases per 100,000 people (10). It is a disease characterized by muscle weakness developing in one side of the face, epiphora, pain in the ear, impaired taste sensation and oversensitivity to sounds. To make the diagnosis of BP, cranial neuropathies, auricular pathologies, cerebellopontin angle pathologies and cranial pathologies, all of which cause paresis/paralysis, should be ruled out (11).

The etiopathogenesis of BP has not yet been completely understood. Infections, genetic factors, microvascular circulatory impairment, immunological factors and inflammation have all been proposed (4). In a study by Yllmaz et al. (4), inflammatory cytokines were compared between BP patients and healthy controls and serum IL-6, IL-8 and TNF-alpha values were found to be significantly higher in patients with BP. They reported that IL-6, IL-8 and TNF-alpha values were not correlated with the degree of improvement in patients with BP.

The neutrophil to lymphocyte ratio (NLR) and PLR can be easily calculated and are cost-effective tests. NLR has been recommended as a new marker for systemic inflammation (6). It has also been shown to be useful in the prediction of mortality, both in the short-term and long-term in acute
TABLE 2. Patients' complete blood count parameters

\begin{tabular}{lccc}
\hline & BP Group & Control Group & p value \\
\hline NLR & $4.37 \pm 3.87$ & $1.89 \pm 0.72$ & $<0.001$ \\
PLR & $137.50 \pm 81.04$ & $113.75 \pm 35.83$ & 0.008 \\
WBC & $11.13 \pm 4.56$ & $7.33 \pm 1.42$ & $<0.001$ \\
Neutrophil & $8.07 \pm 4.24$ & $4.22 \pm 1.07$ & $<0.001$ \\
Lymphocyte & $2.33 \pm 0.98$ & $2.36 \pm 0.59$ & 0.766 \\
Platelet & $267.56 \pm 73.74$ & $256.28 \pm 64.93$ & 0.255 \\
\hline BP: Bell's palsy; NLR: neutrophil to lymphocyte ratio; PLR: platelet to lymphocyte \\
ratio; WBC: leukocyte
\end{tabular}

TABLE 3. The distribution of mean NLR and mean PLR by the patient groups divided according to House-Brackmann Grading of Paralysis

\begin{tabular}{lccccc}
\hline $\begin{array}{l}\text { Paralysis } \\
\text { grade }\end{array}$ & $\begin{array}{c}\text { Number } \\
\text { of } \\
\text { patients }\end{array}$ & $\begin{array}{c}\text { NLR } \\
\text { (mean } \pm \text { standard } \\
\text { deviation) }\end{array}$ & $\begin{array}{c}\text { NLR } \\
\text { p value }\end{array}$ & $\begin{array}{c}\text { PLR } \\
\text { (mean } \pm \text { standard } \\
\text { deviation) }\end{array}$ & $\begin{array}{c}\text { PLR } \\
\text { p value }\end{array}$ \\
\hline 2 & 53 & $4.43 \pm 3.59$ & & $140.38 \pm 81.84$ & \\
3 & 32 & $4.38 \pm 4.45$ & 0.709 & $135.24 \pm 84$ & 0.925 \\
4 & 11 & $4.24 \pm 4.21$ & & $134.77 \pm 79.27$ & \\
5 & 3 & $3.71 \pm 1.48$ & & $122.47 \pm 30.86$ & \\
\hline
\end{tabular}

NLR: neutrophil to lymphocyte ratio; PLR: platelet to lymphocyte ratio

coronary syndrome. In fact, mortality is increased in patients with high NLR (6). In addition, in a study by Ulu et al. (12), NLR was significantly higher in patients with sudden hearing loss than in healthy controls. They also reported that a decrease in response to treatment was observed in patients with high NLR and that high NLR was indicative of poor prognosis.

Bucak et al. (9), in their study of 54 patients with BP and healthy controls, found that the mean neutrophil count was $5.86 \pm 2.38$ and that the mean NLR was $2.69 \pm 1.48$ in the patients, which were significantly higher than the results in the controls. In the present study, the mean neutrophil count was $8.07 \pm 4.24$ in the BP patients, which was significantly higher than in the control group $(\mathrm{p}<0.001)$, and the mean NLR was $4.37 \pm 3.87$ in the BP patients and $1.89 \pm 0.72$ in the control group, with a significant difference $(\mathrm{p}<0.001)$.

As far as we know, there has not been a study comparing the degree of facial paralysis and NLR and PLR. In the present study, most of the patients in the BP group had grade 2 or 3 facial paralysis. No significant relation was detected between the degree of facial paralysis and NLR and PLR.

To conclude, NLR and PLR were significantly higher in patients with BP than in healthy controls. This is the first study to show the relation between BP and PLR. However, no relation was found between the degree of BP and NLR and PLR. It is recommended that NLR and PLR be used as auxiliary tests in the diagnosis of BP since they are inexpensive and can be calculated easily and quickly. 
Ethics Committee Approval: Ethics committee approval was received for this study from the Ethics Committee of Ankara Numune Education and Research Hospital (Approval Number 246/2014).

Informed Consent: Written informed consent was obtained from patients who participated in this study.

Peer-review: Externally peer-reviewed.

Author contributions: Concept - D.A., A.İ., S.K.; Design - D.A, A.İ, K.M.Ö, M.A.Ç.; Supervision - K.M.Ö., S.E., H.D.; Materials - D.A. A.I., S.K., M.A.Ç.; Data Collection \&/or Processing - D.A., K.M.Ö., S.E. Analysis \&/or Interpretation - S.K., M.A.Ç., H.D.; Literature Search - D.A., A.İ., K.M.Ö.; Writing - D.A., S.K., M.A.Ç.; Critical Reviews - K.M.Ö., S.E., H.D.

Conflict of Interest: No conflict of interest was declared by the authors.

Financial Disclosure: The authors declared that this study has received no financial support.

\section{REFERENCES}

1. Bleicher JN, Hamiel S, Gengler JS, Antimarino J. A survey of facial paralysis: etiology and incidence. Ear Nose Throat J 1996;75:355-8.

2. Hughes GB. Practical management of Bell's palsy. Otolaryngol Head Neck Surg 1990;102:658-63.

3. Peitersen E. The natural history of Bell's palsy. Am J Otol 1982;4:107-11.

4. Yilmaz M, Tarakcioglu M, Bayazit N, Bayazit YA, Namiduru M, Kanlikama M. Serum cytokine levels in Bell's palsy. J Neurol Sci 2002;197:69-72. [CrossRef]
5. Kefalidis G, Riga M, Argyropoulou P, Katotomichelakis M, Gouveris C, Prassopoulos P, et al. Is the width of the labyrinthine portion of the fallopian tube implicated in the pathophysiology of Bell's palsy?: a prospective clinical study using computed tomography. Laryngoscope 2010;120:1203-7. [CrossRef]

6. Bhat T, Teli S, Rijal J, Bhat H, Raza M, Khoueiry G, et al. Neutrophil to lymphocyte ratio and cardiovascular diseases: a review. Expert Rev Cardiovasc Ther 2013;11:55-9. [CrossRef]

7. Proctor MJ, McMillan DC, Morrison DS, Fletcher CD, Horgan PG, Clarke SJ. A derived neutrophil to lymphocyte ratio predicts survival in patients with cancer. Br J Cancer 2012;107:695-9. [CrossRef]

8. Wang D, Yang JX, Cao DY, Wan XR, Feng FZ, Huang HF, et al. Preoperative neutrophil-lymphocyte and platelet-lymphocyte ratios as independent predictors of cervical stromal involvement in surgically treated endometrioid adenocarcinoma. Onco Targets Ther 2013;6:211-6.

9. Bucak A, Ulu S, Oruc S, Yucedag F, Tekin MS, Karakaya F, et al. Neutrophil-to-lymphocyte ratio as a novel-potential marker for predicting prognosis of Bell palsy. Laryngoscope 2014;124:1678-81. [CrossRef]

10. Morris AM, Deeks SL, Hill MD, Midroni G, Goldstein WC, Mazzulli T, et al. Annualized incidence and spectrum of illness from an outbreak investigation of Bell's palsy. Neuroepidemiology 2002;21:255-61. [CrossRef]

11. Greco A, Gallo A, Fusconi M, Marinelli C, Macri GF, de Vincentiis M. Bell's palsy and autoimmunity. Autoimmun Rev 2012;12:323-8. [CrossRef]

12. Ulu S, Ulu MS, Bucak A, Ahsen A, Yucedag F, Aycicek A. Neutrophil-to-lymphocyte ratio as a new, quick, and reliable indicator for predicting diagnosis and prognosis of idiopathic sudden sensorineural hearing loss. Otol Neurotol 2013;34:1400-4. [CrossRef] 